\title{
Angioplastia coronaria de arteria circunfleja con arteria coronaria izquierda anómala y nacimiento desde el seno de Valsalva derecho. Reporte de un caso
}

\author{
Coronary angioplasty in coronay left circunflex anomaly. \\ Case report and discussion of literature
}

Rodrigo A. Martín', Ricardo M. Cetera' , Amalia Descalzo', Sergio Zolorza'

\section{RESUMEN}

Presentamos un caso clínico de hallazgo infrecuente en el cual el paciente presenta asociación de arteria coronaria izquierda anómala y lesión aterosclerótica obstructiva sobre la arteria circunfleja. En base a esta condición realizamos una búsqueda sobre la bibliografía existente.

Palabras claves: coronaria anómala, angioplastia coronaria, stent.

\section{ABSTRACT}

We present a clinical case of infrequent finding in which the patient presents association of abnormal left coronary artery and obstructive atherosclerotic injury on the circumflex artery. Based on this condition we carry out a search on the existing bibliography.

Key words: coronary anomaly, coronary angioplasty, stents.

Revista Argentina de Cardioangiología Intervencionista 2020;11(2):86-87. https://doi.org/10.30567/RACI/202002/0086-0087

\section{INTRODUCCIÓN}

El nacimiento de la arteria coronaria izquierda desde el seno de Valsalva derecho corresponde a una anomalía coronaria muy infrecuente, con una incidencia entre el 0,2 y $1,2 \%$. No suele producir repercusión clínica y su diagnóstico suele ser un hallazgo ocasional. Sin embargo, puede asociarse a isquemia miocárdica e incluso muerte súbita especialmente en pacientes jóvenes durante el ejercicio. En caso de existencia de enfermedad aterosclerótica coronaria, esta no suele desarrollarse sobre un trayecto coronario anómalo. Se presenta el caso de un paciente en el que se halló enfermedad obstructiva de arteria circunfleja con nacimiento del tronco de coronaria izquierda a nivel del seno coronario derecho en el que se realizó angioplastia coronaria con stent.

\section{CASO CLÍNICO}

Paciente masculino de 66 años con factores de riesgo cardiovascular positivos para hipertensión arterial, tabaquismo y dislipemia. Presentaba como antecedente un síndrome coronario agudo sin elevación del segmento ST con 2 años de anterioridad por el cual se le realizó angioplastia transluminal coronaria sobre arteria coronaria derecha. El paciente evolucionó asintomático hasta hace 6 meses previo a su consulta, cuando comenzó con ángor en CF II y se le realizó SPECT que evidenció isquemia moderada en territorio lateroapical, anterolateroapical y anterolateromedial. Fue derivado al ser-

\footnotetext{
1. Hospital General de Agudos Presidente Perón. Avellaneda. Servicio de Cardiología Intervencionista y Hemodinamia.

$\triangle$ Correspondencia:Rodrigo A. Martin. Juan B. Alberdi 4296.CABA. roanmartin@ hotmail.com

Los autores declaran no tener conflictos de intereses.
}

Recibido: 21/07/2019|Aceptado: 14/05/2020 vicio de Cardiología Intervencionista para intervención coronaria percutánea. Se realizó cinecoronariografía por acceso femoral con introductor Super Sheath (Boston Scientific) 6 Fr y catéteres diagnósticos 6 Fr Judkins Left 3.5 y Judkins Right 3 Impulse (Boston Scientific), hallándose a la arteria coronaria derecha sin lesiones angiográficas significativas y se observa desde el mismo seno coronariano derecho el nacimiento del tronco de la arteria coronaria izquierda. A este nivel puede observarse la presencia de una obstrucción significativa a partir del segmento medio de la arteria circunfleja (Figuras 1 y 2). En forma programada (Figuras 3 y 4 ) y por acceso femoral con introductor Super Sheath 6 Fr y catéter guía Convey Left 6 Fr (Boston Scientific) se realizó a través de cuerda guía Choice Floppy 0,014x180 (Boston Scientific), la cual con dificultad por severa tortuosidad se posicionó en el segmento distal de la arteria circunfleja, dilatación con balón coronario Emerge 2,5x15 mm (Boston Scientific) e implante final de stent coronario liberador de everolimus de 3,0x16 mm tipo Promus Premier (Boston Scientific).

\section{DISCUSIÓN}

Las anomalías de las arterias coronarias identificadas por cinecoronariografía son hallazgos no habituales ${ }^{1}$, dentro de las cuales el origen de la arteria coronaria izquierda en el seno de Valsalva derecho constituye un patrón muy raro con una incidencia entre el 0,2 y 1,2\% según la serie ${ }^{5}$. Los pacientes portadores de esta anomalía suelen cursar clínicamente asintomáticos, con la excepción que el trayecto anómalo discurra entre la aorta y la arteria pulmonar, lo que puede ocasionar muerte súbita en especial durante la actividad física ${ }^{2}$. La incidencia de síntomas graves es de aproximadamente el $20 \%$.

La asociación entre esta anomalía y enfermedad aterosclerótica coronaria no es un hecho común; sin embargo, cuando existen, rara vez afectan el segmento anómalo. En 


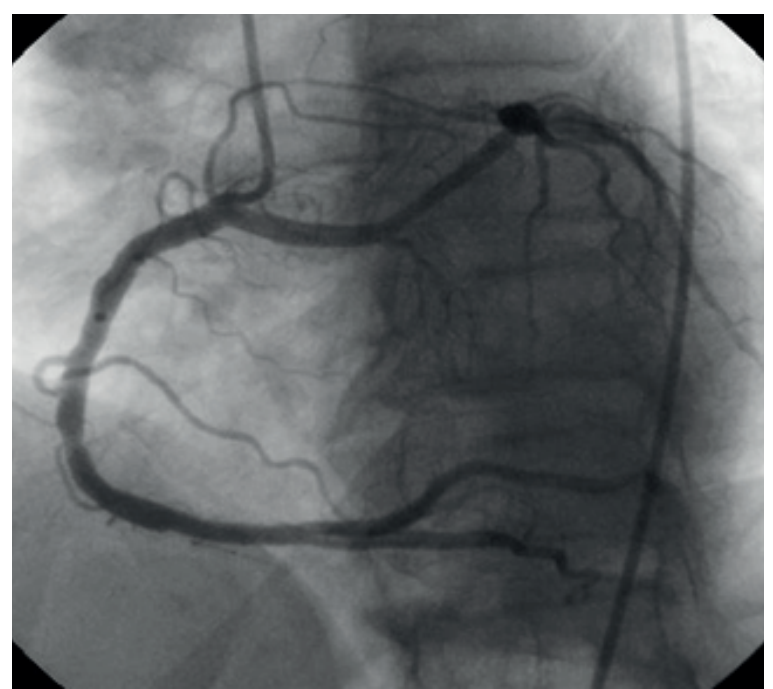

Figura 1. Se observa, durante la canalización de la arteria coronaria derecha, el nacimiento de la arteria coronaria izquierda en el mismo seno.

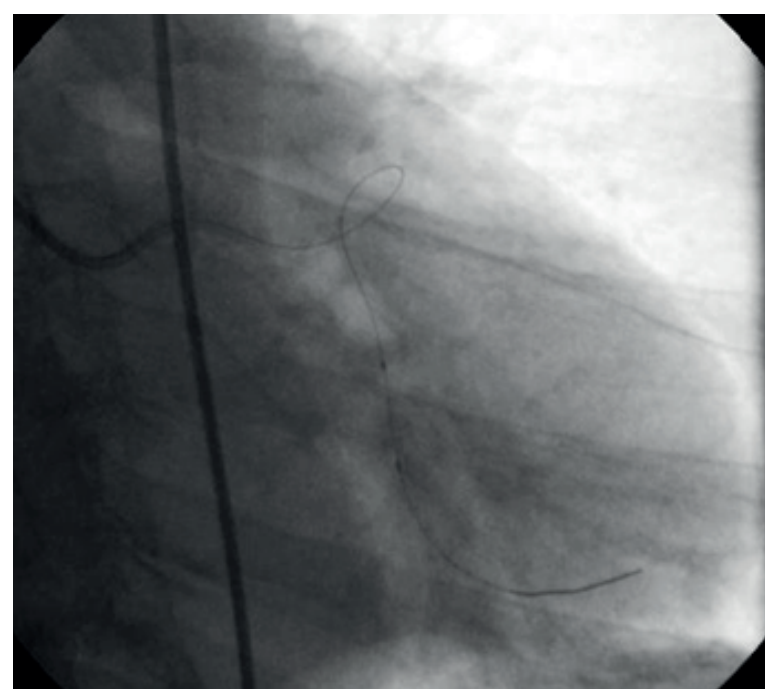

Figura 3. Cruce de lesión con cuerda Floppy 0,014 predilatación con balón coronario 2,5×15 mm e implante de stent farmacoactivo $3,0 \times 16 \mathrm{~mm}$.

el caso de presencia de placa aterosclerótica obstructiva sobre el territorio anómalo, esta se desarrolla más agresivamente y en estadios más tempranos desde el punto de vista fisiopatológico ${ }^{3}$.

La angiografía coronaria es el método de elección para la identificación de lesiones ateroscleróticas ${ }^{4}$; sin embargo, puede causar algunos problemas diagnósticos en la identificación tridimensional del curso de la arteria anómala. En este sentido, la tomografía computarizada multicortes (TCM) ayuda a una

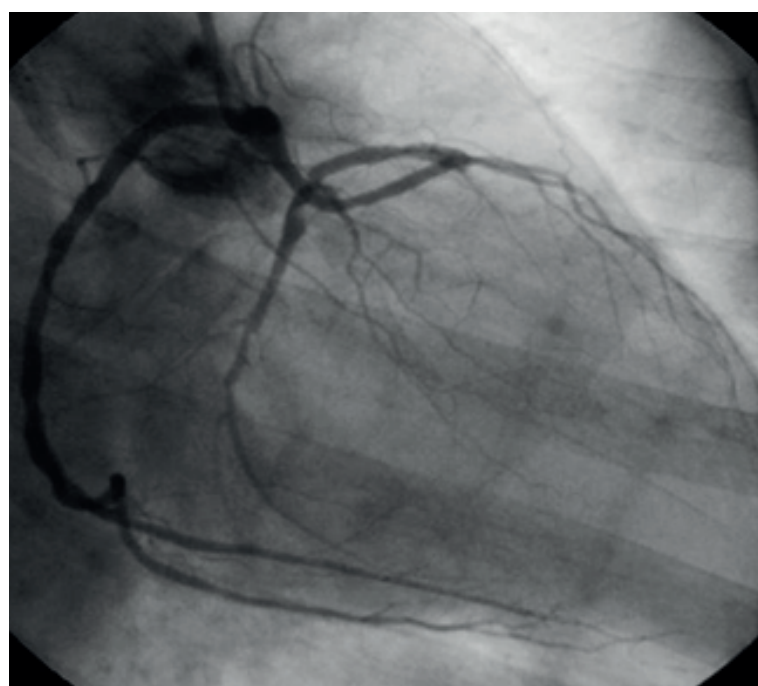

Figura 2. Identificación de lesión significativa a nivel del segmento distal de la arteria circunfleja anómala.

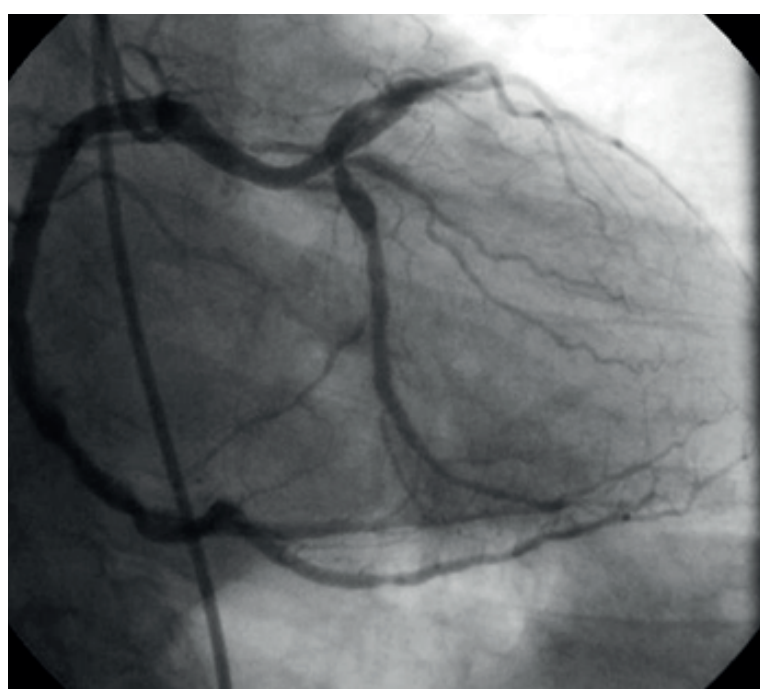

Figura 4. Resultado final, con resolución de la lesión preexistente. No se observa angiográficamente lesión residual.

mejor visualización de la porción proximal, tamaño, curso y relación del vaso anómalo con las estructuras circundantes.

Nuestro caso se refiere a un paciente en la sexta década de la vida en el cual se considera un trayecto anómalo posiblemente benigno, con antecedentes ya demostrados de enfermedad aterosclerótica sobre coronaria derecha y con estudios complementarios que evidenciaban la presencia de isquemia. Debido a estas condiciones se decidió realizar angioplastia coronaria sobre la lesión encontrada ${ }^{6}$.

\section{BIBLIOGRAFÍA}

1. Angelini P, Velasco JA, Flamm S. Coronary anomalies: incidence, pathophysiology, and clinical relevance. Circulation 2002; 105: 2449-2454.

2. Chaitman BR, Lesperance J, Saltiel J, et al. Clinical, angiographic, and hemodynamic findings in patients with anomalous origin of the coronary arteries. Circulation 1976;53: 122-131.

3. Click RL, Holmes DR, Jr, Vlietstra RE, et al. Anomalous coronary arteries: location, degree of atherosclerosis and effect on survival: a report from the Coronary Artery Surgery Study. J Am Coll Cardiol 1989; 13: 531-37.
4. Gersony WM. Management of anomalous coronary artery from the contralateral coronary sinus. J Am Coll Cardiol 2007; 50:2083-4.

5. Ugalde H, Ramírez A, Ugalde D, Farías E, Silva AM. Nacimiento anómalo del arterias coronarias en 10.000 pacientes adultos sometidos a coronariografía. Rev Med Chil 2010; 138: 7-14.

6. Grasso AE, Pennell DJ. Myocardial infarction related to aberrant left circumflex artery, Int J Cardiol 2010; 138: 51-52. 